\title{
CONVERGENCE OF EXPONENTIAL ATTRACTORS FOR A FINITE ELEMENT APPROXIMATION OF THE ALLEN-CAHN EQUATION
}

\author{
MORGAN PIERRE
}

\begin{abstract}
We consider a space semidiscretization of the Allen-Cahn equation by conforming Lagrange finite elements. For every mesh parameter $h$, we build an exponential attractor $\mathcal{M}_{h}$ of the dynamical system associated to the approximate equations. We prove that, as $h$ tends to $0, \mathcal{M}_{h}$ converges for the symmetric Hausdorff distance to an exponential attractor $\mathcal{M}_{0}$ of the dynamical system associated to the Allen-Cahn equation. We also provide an explicit estimate of this distance and we prove that the fractal dimension of $\mathcal{M}_{h}$ and of the global attractor is bounded by a constant independent of $h$. Our proof is adapted from the result of Efendiev, Miranville and Zelik concerning the continuity of exponential attractors under perturbation of the underlying semigroup. Here, for the first time, the perturbation is a space discretization. The case of a time semidiscretization has been analyzed in a previous paper.
\end{abstract}

Keywords: Allen-Cahn equation, finite element method, global attractor, exponential attractor.

\section{INTRODUCTION}

In this paper, we consider a space semidiscretization of the Allen-Cahn equation by conforming Lagrange $\mathbb{P}_{k}$ finite elements $(k \geq 1)$. We build a family of exponential attractors associated to the discretized equations which is robust as the mesh parameter $h$ tends to 0 .

For a dissipative dynamical system, an exponential attractor is a compact positively invariant set which contains the global attractor, has finite fractal dimension and attracts exponentially the trajectories. In comparison with the global attractor, an exponential attractor is expected to be more robust to perturbations: global attractors are generally upper semicontinuous with respect to perturbations, but the lower semicontinuity can be proved only in some particular cases (see e.g. $[2,18,20,24])$. This includes perturbations which are obtained by time and/or space discretization of the governing equations [23, 26, 27]). We can also note that an exponential attractor is generally not unique, in contrast with the global attractor.

In the initial construction proposed by Eden et al. [5], based on a "squeezing property", the continuity of exponential attractors was shown for classical Galerkin approximations, but only up to a time shift (see also $[11,13]$ ). Related robustness results were also obtained in [1] for finite element approximations. In [7], Efendiev, Miranville and Zelik proposed a construction of exponential attractors based on a "smoothing property" and on an appropriate error estimate, where the continuity holds without time shift. Their construction has been adapted to many situations, including singular perturbations. We refer the reader to the review [18] and the references therein for more details. 
In [19], the author used the result of Efendiev, Miranville and Zelig to analyze the case where the perturbation is a time semidiscretization of the underlying equation, and when the time step goes to 0 . An abstract construction of a robust family of exponential attractors was first proposed, and then applied in every space dimension to the backward Euler time semidiscretization of the Allen-Cahn equation with a polynomial nonlinearity. It was also applied in [3] to the case of a time-splitting discretization of the Caginalp phase-field system.

The purpose of this paper is to address the case of a space semidiscretization for a simple model problem. We consider the Allen-Cahn equation on a bounded and convex domain of $\mathbb{R}^{d}(d=1,2$ or 3$)$ with a polynomial nonlinearity. We use Dirichlet boundary conditions and we impose a growth restriction in space dimension 3 . This allows us to work with an absorbing set in the $H_{0}^{1}$ Sobolev space and it avoids using $L^{\infty}$ estimates which would be delicate to obtain.

Two key ingredients in our proof are a $L^{2}-H^{1}$ smoothing property and a (rather) standard $L^{2}$ error estimate with nonsmooth data $[8,15]$. However, in contrast to the time semidiscrete case, we cannot apply directly the robustness result in [7] and instead, we adapt the construction, by borrowing some ideas from the singularly perturbed case $[12,17]$. We also use that the space discretization is conforming, i.e. the finite element space is a subspace of $H_{0}^{1}$. We first build a robust family of exponential attractors indexed by $h$ and associated to an appropriate family of discrete-in-time dynamical systems (Lemma 5.4). As a consequence, we obtain an upper bound on the fractal dimension of the global attractors which is independent of $h$ (Theorem 5.5). For these two results, we only assume that the family of triangulations of the domain is regular.

For the Allen-Cahn equation, the upper semicontinuity of the global attractor with respect to the discretization parameters was shown in [22] (see also [9] for existence results with several discretizations). But, to the best of our knowledge, our upper bound on the fractal dimension is a new result. It is a crude bound, but it can be written explicitly in terms of the parameters of the problem. A similar bound was shown in [19] for the time semidiscrete case. In contrast, only a few authors have obtained upper bounds which are independent of the discretization parameters (see e.g. $[28,29])$.

In our main result, Theorem 6.3, we build for every mesh parameter $h$ an exponential attractor $\mathcal{M}_{h}$ associated to the space semidiscrete Allen-Cahn equation. We prove that, as $h$ tends to $0, \mathcal{M}_{h}$ converges for the symmetric Hausdorff distance to an exponential attractor $\mathcal{M}_{0}$ of the Allen-Cahn equation. We also prove that the fractal dimension of $\mathcal{M}_{h}$ is bounded by a constant independent of $h$. This result is based on the the previous construction, but we need the (stronger) assumption that the family of triangulations is quasi-uniform, because we introduce an inverse estimate.

To conclude, we point out that, by combining the time semidiscrete case in [19] and the space semidiscrete approach developed here, we can build similarly a robust family of exponential attractors for a fully discrete approximation of the Allen-Cahn equation. In order to generalize the construction, it would be interesting to investigate other problems with the same approach. For instance, it could be interesting to work on the Cahn-Hilliard equation, the two-dimensional Navier-Stokes equation, or the complex Ginzburg-Landau equation with other choices of space discretization (finite difference, finite volume, ....). 
The paper is organized as follows. First, we give the a priori estimates for the continuous problem (Section 2). Then we introduce the space discrete problem and we show the discrete counterparts of the a priori estimates (Section 3). In Section 4, we establish the error estimate with nonsmooth data. In Section 5, we present our intermediate construction of a robust family of exponential attractors and its application to the global attractor. In the last section, we prove our main result.

\section{The Continuous PROBlem}

2.1. The continuous semigroup $S_{0}$. We consider the following reaction-diffusion equation

$$
\partial_{t} u-\Delta u+g(u)=0 \quad x \in \Omega, t>0,
$$

subject to homogeneous Dirichlet boundary conditions. Here, $\Omega$ is an open bounded subset of $\mathbb{R}^{d}(d \in\{1,2,3\})$ with a $C^{2}$ boundary $\Gamma$. In order to deal with conforming finite elements in the next section, we also assume that $\Omega$ is convex. The nonlinearity $g$ is a polynomial of odd degree with a positive leading coefficient,

$$
g(s)=\sum_{j=0}^{2 p-1} b_{j} s^{j}, \quad b_{2 p-1}>0, p \geq 1 .
$$

If $d=3$, we assume that $p \in\{1,2\}$ (no restriction on $p$ if $d=1$ or $d=2$ ). The restriction on $p$ allows us to use a $H^{1}$ setting; it avoids the use of $L^{\infty}$ estimates. Note that equation (2.1) is linear if and only if $p=1$. When $g(s)=s^{3}-s$ (in which case $p=2$ ), equation (2.1) is known as the Allen-Cahn equation.

We supplement (2.1) with an initial condition

$$
u(0)=u_{0} .
$$

We set $H=L^{2}(\Omega)$ with norm $|\cdot|_{H}$ and scalar product $(\cdot, \cdot)_{H}$. We denote $V=H_{0}^{1}(\Omega)$ with norm $\|\cdot\|_{V}=|\nabla \cdot|_{L^{2}(\Omega)^{d}}$. The scalar product in $L^{2}(\Omega)^{d}$ will be written $(\cdot, \cdot)_{0}$. We denote $\mathbb{R}_{+}$the interval $[0,+\infty)$. For an nonempty interval $I$ of $\mathbb{R}$ and for a Banach space $E$, we denote $C^{0}(I ; E)$ the space of functions which are continuous on $I$ with values in $E$; for $q \geq 1, L^{q}(I ; E)$ is the usual Banach space of (classes of) functions endowed with the norm $v \mapsto\left(\int_{I}\|v(t)\|_{E}^{q} d t\right)^{1 / q}$. The norm in $L^{q}(\Omega)$ is denoted $\|\cdot\|_{L^{q}}$. Throughout the paper, $C$ denotes a generic constant whose dependence on one or several appropriate parameters will be specified.

The following existence and uniqueness result is well-known (see e.g. [16, 24]).

Theorem 2.1. For $u_{0} \in H$, there exists a unique solution $u$ of (2.1)-(2.2) which satisfies $u \in C^{0}\left(\mathbb{R}_{+} ; H\right)$ and $u \in L^{2}(0, T ; V) \cap L^{2 p}\left(0, T ; L^{2 p}(\Omega)\right)$, for all $T>0$. For all $t \geq 0$, the mapping $u_{0} \mapsto u(t)$ is continuous in $H$. If, furthermore, $u_{0} \in V$, then $u$ belongs to $C^{0}([0, T] ; V) \cap L^{2}\left(0, T ; H^{2}(\Omega)\right)$, for all $T>0$.

This result allows to define the continuous-in-time semigroup $S_{0}$ (cf. Section 5.1):

$$
S_{0}(t): u_{0} \in H \mapsto u(t) \in H .
$$

2.2. Some useful inequalities. First recall the Poincaré inequality: there exists a constant $c_{0}=c_{0}(d, \Omega)$ such that

$$
|w|_{H} \leq c_{0}\|w\|_{V}, \quad \forall w \in V .
$$


More generally, the Sobolev imbedding $V \subset L^{q}(\Omega)$ holds for every $q \in[1,+\infty)$ if $d=1$ or $d=2$ and for every $q \in[1,6]$ if $d=3$. The assumption on $p$ guarantees that $g$ is Lipschitz continuous from bounded sets of $V$ into $H$.

Next, we collect a few inequalities related to $g$. Since $\sum_{j=1}^{2 p-2} j b_{j} s^{j-1}$ is a polynomial of degree less than $s^{2 p-2}$, there exists a constant $c_{1}^{\prime}>0$ such that

$$
\left|\sum_{j=1}^{2 p-2} j b_{j} s^{j-1}\right| \leq \frac{1}{2}(2 p-1) b_{2 p-1} s^{2 p-2}+c_{1}^{\prime}, \quad \forall s \in \mathbb{R} .
$$

Thus, $g^{\prime}(s)=\sum_{j=1}^{2 p-1} j b_{j} s^{j-1}$ satisfies

$$
\left|g^{\prime}(s)\right| \leq \frac{3}{2}(2 p-1) b_{2 p-1} s^{2 p-2}+c_{1}^{\prime}, \quad \forall s \in \mathbb{R},
$$

and

$$
\frac{2 p-1}{2} b_{2 p-1} s^{2 p-2}-c_{1}^{\prime} \leq g^{\prime}(s) \leq \frac{3}{2}(2 p-1) b_{2 p-1} s^{2 p-2}+c_{1}^{\prime}, \quad \forall s \in \mathbb{R} .
$$

By the mean value theorem we have, for all $s_{1}, s_{2} \in \mathbb{R}$,

$$
\left(g\left(s_{1}\right)-g\left(s_{2}\right)\right)\left(s_{1}-s_{2}\right)=g^{\prime}\left(\xi_{s_{1}, s_{2}}\right)\left(s_{1}-s_{2}\right)^{2} \geq-c_{1}^{\prime}\left(s_{1}-s_{2}\right)^{2},
$$

where $\xi_{s_{1}, s_{2}} \in \mathbb{R}$. We denote

$$
G(s)=\int_{0}^{s} g(\tau) d \tau=\sum_{j=0}^{2 p-1} b_{j} s^{j+1} /(j+1)
$$

an anti-derivative of $g$. Using a similar argument, we have

$$
\frac{1}{4 p} b_{2 p-1} s^{2 p}-c_{0}^{\prime} \leq G(s) \leq \frac{3}{4 p} b_{2 p-1} s^{2 p}+c_{0}^{\prime}, \quad \forall s \in \mathbb{R},
$$

for some constant $c_{0}^{\prime}>0$. There is also a constant $c_{2}^{\prime}>0$ such that

$$
\frac{1}{2} b_{2 p-1} s^{2 p}-c_{2}^{\prime} \leq g(s) s \leq \frac{3}{2} b_{2 p-1} s^{2 p}+c_{2}^{\prime}, \quad \forall s \in \mathbb{R} .
$$

The following lemma is standard (see e.g. [19]).

Lemma 2.2. Let $w_{1}, w_{2} \in V$ with $\left\|w_{i}\right\|_{V} \leq R_{1}(i=1,2)$ and $w_{3} \in H$. Then

$$
\int_{\Omega}\left|g\left(w_{1}\right)-g\left(w_{2}\right)\right|\left|w_{3}\right| d x \leq C\left(R_{1}\right)|| w_{1}-w_{2} \|_{V}\left|w_{3}\right|_{H},
$$

where $C\left(R_{1}\right)$ is monotonic in $R_{1}$.

2.3. A priori estimates for the solution. In Section $2.3, u$ denotes a solution of (2.1)-(2.2). Propositions 2.3 and 2.4 are proved in [24].

Proposition 2.3 (Absorbing set in $H$ ). There exist a constant $\mathcal{R}_{0}>0$ and a monotonic function $\mathcal{T}_{0}(\cdot)$ such that for all $u_{0} \in H$,

$$
|u(t)|_{H} \leq \mathcal{R}_{0}, \quad \forall t \geq \mathcal{T}_{0}\left(\left|u_{0}\right|_{H}\right) .
$$

In the remainder of the paper, $r>0$ denotes an arbitrary (but fixed) real number.

Proposition 2.4 (Absorbing set in $V$ ). There exists a constant $\mathcal{R}_{1}>0$ such that for all $u_{0} \in H$,

$$
\|u(t)\|_{V} \leq \mathcal{R}_{1}, \quad \forall t \geq \mathcal{T}_{0}\left(\left|u_{0}\right|_{H}\right)+r .
$$


Using the gradient flow structure of the equation, we have (see e.g. [19]):

Lemma 2.5. For any $R_{1}>0$, there exists a constant $C_{1}\left(R_{1}\right)$ such that for all $u_{0} \in V$ with $\left\|u_{0}\right\|_{V} \leq R_{1}$,

$$
\|u(t)\|_{V}^{2}+\int_{0}^{t}\left|\partial_{t} u\right|_{H}^{2} d s \leq C_{1}\left(R_{1}\right), \quad \forall t \geq 0 .
$$

In particular, for all $t_{1}, t_{2} \geq 0$, we have

$$
\left|u\left(t_{1}\right)-u\left(t_{2}\right)\right|_{H}^{2} \leq C_{1}\left(R_{1}\right)\left|t_{1}-t_{2}\right| .
$$

2.4. Estimates for the difference of solutions. In the following lemmas, $u$ and $\hat{u}$ denote two solutions of $(2.1)$, and $v(t)=u(t)-\hat{u}(t)$ is their difference, which satisfies

$$
\partial_{t} v-\Delta v+g(u)-g(\hat{u})=0 \quad \text { in } \Omega \times \mathbb{R}_{+} .
$$

The following two lemmas are standard (see e.g. [19]).

Lemma 2.6. For all $t \geq 0$,

$$
|v(t)|_{H}^{2}+2 \int_{0}^{t}\|v\|_{V}^{2} d s \leq|v(0)|_{H}^{2} \exp \left(2 c_{1}^{\prime} t\right) .
$$

The following result shows a smoothing property [18].

Lemma 2.7. If $\|u(0)\|_{V} \leq R_{1}$ and $\|\hat{u}(0)\|_{V} \leq R_{1}$, then for all $t>0$, we have

$$
\|v(t)\|_{V}^{2} \leq C_{2}\left(R_{1}, t\right)|v(0)|_{H}^{2}
$$

where the function $C_{2}:(0,+\infty)^{2} \rightarrow \mathbb{R}_{+}$is continuous.

\section{The SPACE SEMidisCRETE PROBLEM}

The estimates for the space semidiscrete problem are analogous to those of the continuous problem. For the reader's convenience, we will sketch the proofs. We first describe the finite element approximation.

3.1. Definition of the finite element space. We use continuous Lagrange $\mathbb{P}_{k}$ finite element which are $H_{0}^{1}$ conforming (cf. (3.1)). We do not need isoparametric elements, because low order errors are sufficient for our purpose (cf. (4.1)). Here, $k \geq 1$ is an integer which is fixed in the remainder of the paper.

Recall that $\Omega$ is a bounded and convex open subset of $\mathbb{R}^{d}$ with smooth boundary. Following [21, Section 5.2], we approximate $\Omega$ by a convex open $d$-polyhedra $\Omega_{h}$ included in $\Omega$ (i.e. an interval if $d=1$, a convex polygon if $d=2$, and a convex polyhedra if $d=3$ ), and where the vertices of the boundary $\Gamma_{h}$ of $\Omega_{h}$ lie on the boundary $\Gamma$ of $\Omega$.

We denote $\mathcal{T}_{h}$ a triangulation of $\bar{\Omega}_{h}$ into closed $d$-simplices $K$. For every $d$-simplex $K \in \mathcal{T}_{h}$, we let $h_{K}$ be the diameter of $K$ and $\rho_{K}$ be the diameter of its inscribed $d$-ball. As usually, we denote $h=\max _{K \in \mathcal{T}_{h}} h_{K}$. In our study as $h \rightarrow 0$, we assume that we have a regular family $\left(\mathcal{T}_{h}\right)_{h \in J}$ of such triangulations, which means that there exists a constant $\sigma_{0}>0$ such that $[10]$

$$
\forall h \in J, \forall K \in \mathcal{T}_{h}, \quad \frac{h_{K}}{\rho_{K}} \leq \sigma_{0}
$$


Here, $J$ is a subset of a bounded interval $\left(0, h_{\max }\right]$ which admits 0 as a limit point. Since $\Omega$ has a smooth boundary $\Gamma$, there is a constant $C_{\Gamma}<+\infty$ such that

$$
\forall h \in J, \forall K \in \mathcal{T}_{h}, \forall x \in \Gamma_{h} \cap K, \quad \operatorname{dist}(x, \Gamma) \leq C_{\Gamma} h_{K}^{2} .
$$

For a given $\mathcal{T}_{h}$, the associated finite element space is

$$
V_{h}=\left\{v \in C^{0}(\bar{\Omega}): v=0 \text { on } \bar{\Omega} \backslash \Omega_{h} \text { and } \forall K \in \mathcal{T}_{h},\left.v\right|_{K} \in \mathbb{P}_{k}\right\},
$$

where $\mathbb{P}_{k}$ is the space of polynomials in $d$ variables of order less than or equal to $k$. Since $\bigcup_{K \in \mathcal{T}_{h}} K=\bar{\Omega}_{h} \subset \bar{\Omega}$, the space $V_{h}$ is well defined, and it is well known that

$$
V_{h} \subset H_{0}^{1}\left(\Omega_{h}\right) \subset H_{0}^{1}(\Omega)=V .
$$

3.2. The semigroup $S_{h}$. The space semidiscrete scheme reads: find $u_{h}: \mathbb{R}_{+} \rightarrow V_{h}$ such that

$$
\frac{d}{d t}\left(u_{h}(t), \varphi_{h}\right)_{H}+\left(\nabla u_{h}(t), \nabla \varphi_{h}\right)_{0}+\left(g\left(u_{h}(t)\right), \varphi_{h}\right)_{H}=0, \quad \forall t \geq 0, \forall \varphi_{h} \in V_{h},
$$

together with the initial condition

$$
u_{h}(0)=u_{h}^{0}
$$

where $u_{h}^{0}$ is given in $V_{h}$.

Since $V_{h}$ has finite dimension, it is easily seen that for every $u_{h}^{0} \in V_{h}$, problem (3.2)(3.3) has a unique solution $u_{h} \in C^{1}\left(\mathbb{R}_{+}, V_{h}\right.$ ) (see e.g. [9]). Thus, the semigroup $S_{h}$ acting on $V_{h}$ is well-defined:

$$
S_{h}(t): u_{h}^{0} \in V_{h} \mapsto u_{h}(t) \in V_{h} .
$$

3.3. A priori estimates for the solution, uniform in $h$. In Section 3.3, $u_{h}$ denotes a solution of (3.2)-(3.3).

Proposition 3.1 (Absorbing set for the $H$-norm). For all $u_{h}^{0} \in V_{h}$,

$$
\left|u_{h}(t)\right|_{H} \leq \mathcal{R}_{0}, \quad \forall t \geq \mathcal{T}_{0}\left(\left|u_{h}^{0}\right|_{H}\right),
$$

where the constant $\mathcal{R}_{0}$ and the monotonic function $\mathcal{T}_{0}(\cdot)$ are independent of $h$ and can be chosen as in Proposition 2.3.

Proof. We choose $\varphi_{h}=u_{h}(t)$ in (3.2) and we use (2.8). This yields

$$
\frac{d}{d t}\left|u_{h}\right|_{H}^{2}+2\left\|u_{h}\right\|_{V}^{2}+b_{2 p-1}\left(u_{h}^{2 p}, 1\right)_{H} \leq 2 c_{2}^{\prime}|\Omega|, \quad \forall t \geq 0,
$$

where $|\Omega|$ is the Lebesgue measure of $\Omega$. Using the Poincaré inequality (2.3) and the classical Gronwall lemma, we find

$$
\left|u_{h}(t)\right|_{H}^{2} \leq\left|u_{h}^{0}\right|_{H}^{2} \exp \left(-\frac{2 t}{c_{0}^{2}}\right)+\frac{c_{2}^{\prime \prime} c_{0}^{2}}{2}, \quad \forall t \geq 0,
$$

with $c_{2}^{\prime \prime}=2 c_{2}^{\prime}|\Omega|$. This estimate implies the assertion with, for instance, $\mathcal{R}_{0}^{2}=$ $c_{2}^{\prime \prime} c_{0}^{2}$.

Recall that $r>0$ is a fixed real number.

Proposition 3.2 (Absorbing set for the $V$-norm). For all $u_{h}^{0} \in V_{h}$,

$$
\left\|u_{h}(t)\right\|_{V} \leq \mathcal{R}_{1}, \quad \text { if } t \geq \mathcal{T}_{0}\left(\left|u_{h}^{0}\right|_{H}\right)+r,
$$

where the constant $\mathcal{R}_{1}$, independent of $h$, can be chosen as in Proposition 2.4. 
Proof. By integrating (3.4) over $[t, t+r]$, we first obtain $\left|u_{h}(t+r)\right|_{H}^{2}+2 \int_{t}^{t+r}\left\|u_{h}\right\|_{V}^{2} d s+b_{2 p-1} \int_{t}^{t+r}\left(u_{h}^{2 p}, 1\right)_{H} d s \leq\left|u_{h}(t)\right|_{H}^{2}+r c_{2}^{\prime \prime}, \quad \forall t \geq 0$. By (2.7), we have

$$
0 \leq \frac{4 p}{3}\left(G(s)+c_{0}^{\prime}\right) \leq b_{2 p-1} s^{2 p}+c_{0}^{\prime \prime}, \quad \forall s \in \mathbb{R},
$$

where $c_{0}^{\prime \prime}=8 p c_{0}^{\prime} / 3$. For $t \geq \mathcal{T}_{0}\left(\left|u_{h}^{0}\right|_{H}\right)$, we know by the previous proposition that $\left|u_{h}(t)\right|_{H} \leq \mathcal{R}_{0}$, and so

$$
c_{3}^{\prime \prime} \int_{t}^{t+r}\left(\frac{1}{2}\left\|u_{h}(s)\right\|_{V}^{2}+\left(\tilde{G}\left(u_{h}(s)\right), 1\right)_{H}\right) d s \leq \mathcal{R}_{0}^{2}+r c_{2}^{\prime \prime}+r c_{0}^{\prime \prime},
$$

where $c_{3}^{\prime \prime}=\min \{4,4 p / 3\}>0$ and $\tilde{G}(s)=G(s)+c_{0}^{\prime \prime} \geq 0$.

Next, we choose $\varphi_{h}=\frac{d u_{h}}{d t}(t)$ in $(3.2)$, and we find

$$
\left.\left|\frac{d u_{h}}{d t}(t)\right|_{H}^{2}+\frac{d}{d t}\left(\frac{1}{2}\left\|u_{h}(t)\right\|_{V}^{2}+\tilde{G}\left(u_{h}(t)\right), 1\right)_{H}\right)=0, \quad \forall t \geq 0 .
$$

The uniform Gronwall lemma [24] shows that

$$
\left.\frac{1}{2}\left\|u_{h}(t)\right\|_{V}^{2}+\tilde{G}\left(u_{h}(t)\right), 1\right)_{H} \leq \frac{\mathcal{R}_{0}^{2}+r c_{2}^{\prime \prime}+r c_{0}^{\prime \prime}}{r c_{3}^{\prime \prime}} \exp (r), \quad \forall t \geq \mathcal{T}_{0}\left(\left|u_{h}^{0}\right|_{H}\right)+r .
$$

This proves the claim.

By integrating $(3.5)$ on $[0, t]$, we also find:

Lemma 3.3. For any $R_{1}>0$ and for all $u_{h}^{0} \in V$ such that $\left\|u_{h}^{0}\right\|_{V} \leq R_{1}$, we have

$$
\left\|u_{h}(t)\right\|_{V}^{2}+\int_{0}^{t}\left|\frac{d u_{h}}{d t}(s)\right|_{H}^{2} d s \leq C_{1}\left(R_{1}\right), \quad \forall t \geq 0,
$$

where $C_{1}\left(R_{1}\right)$ is the same constant (independent of $h$ ) as in Lemma 2.5. In particular, for all $t_{1}, t_{2} \geq 0$, we have

$$
\left|u_{h}\left(t_{1}\right)-u_{h}\left(t_{2}\right)\right|_{H}^{2} \leq C_{1}\left(R_{1}\right)\left|t_{1}-t_{2}\right| .
$$

3.4. Estimates for the difference of solutions, uniform in $h$. In Section 3.4, $u_{h}$ and $\hat{u}_{h}$ are two solutions of $(3.2)$, and $v_{h}(t)=u_{h}(t)-\hat{u}_{h}(t)$ is their difference, which satisfies

$$
\frac{d}{d t}\left(v_{h}(t), \varphi_{h}\right)_{H}+\left(\nabla v_{h}(t), \nabla \varphi_{h}\right)_{0}+\left(g\left(u_{h}(t)\right)-g\left(\hat{u}_{h}(t)\right), \varphi_{h}\right)_{H}=0, \quad \forall t \geq 0,
$$

for all $\varphi_{h} \in V_{h}$. We first have:

Lemma 3.4. For all $t \geq 0$,

$$
\left|v_{h}(t)\right|_{H}^{2}+2 \int_{0}^{t}\left\|v_{h}(s)\right\|_{V}^{2} d s \leq\left|v_{h}(0)\right|_{H}^{2} \exp \left(2 c_{1}^{\prime} t\right) .
$$

Proof. We choose $\varphi_{h}=v_{h}(t)$ in (3.6) and we use (2.4). We find

$$
\frac{d}{d t}\left|v_{h}\right|_{H}^{2}+2\left\|v_{h}\right\|_{V}^{2} \leq 2 c_{1}^{\prime}\left|v_{h}\right|_{H}^{2} .
$$

The classical Gronwall lemma concludes the proof.

Next, we show a smoothing property, uniform in $h$. 
Lemma 3.5. If $\left\|u_{h}(0)\right\|_{V} \leq R_{1}$ and $\left\|\hat{u}_{h}(0)\right\|_{V} \leq R_{1}$, then for all $t>0$, we have

$$
\left\|v_{h}(t)\right\|_{V}^{2} \leq C_{2}\left(R_{1}, t\right)\left|v_{h}(0)\right|_{H}^{2},
$$

where the continuous function $C_{2}:(0,+\infty)^{2} \rightarrow \mathbb{R}_{+}$, independent of $h$, is the same as in Lemma 2.7.

Proof. From Lemma 3.3, we know that

$$
\left\|u_{h}(t)\right\|_{V}^{2} \leq C_{1}\left(R_{1}\right) \quad \text { and } \quad\left\|\hat{u}_{h}(t)\right\|_{V}^{2} \leq C_{1}\left(R_{1}\right), \quad \forall t \geq 0 .
$$

In (3.6), we choose $\varphi_{h}=\frac{d v_{h}}{d t}(t)$, we apply Lemma 2.2 and Young's inequality. We find

$$
\frac{d}{d t}\left\|v_{h}(t)\right\|_{V}^{2} \leq C\left(R_{1}\right)\left\|v_{h}(t)\right\|_{V}^{2}, \quad t \geq 0
$$

where $C\left(R_{1}\right)$ is independent of $h$. On multiplying by $t$ and adding $\left\|v_{h}(t)\right\|_{V}^{2}$, this yields

$$
\frac{d}{d t}\left(t\left\|v_{h}(t)\right\|_{V}^{2}\right) \leq C\left(R_{1}\right)\left(t\left\|v_{h}(t)\right\|_{V}^{2}\right)+\left\|v_{h}(t)\right\|_{V}^{2}, \quad t \geq 0 .
$$

The classical Gronwall lemma yields

$$
t\left\|v_{h}(t)\right\|_{V}^{2} \leq \exp \left(C\left(R_{1}\right) t\right) \int_{0}^{t}\left\|v_{h}(s)\right\|_{V}^{2} d s, \quad \forall t \geq 0 .
$$

By applying Lemma 3.4, we see that Lemma 3.5 holds with

$$
C_{2}\left(R_{1}, t\right)=\frac{1}{2 t} \exp \left(2 c_{1}^{\prime} t\right) \exp \left(C\left(R_{1}\right) t\right) .
$$

\section{NONSMOOTh DATA ERROR ESTIMATE ON FINITE TIME INTERVALS}

For the error estimate with $H^{1}$ data, we follow the approach in [15]. However, in the paper [15], the nonlinearity $g$ was assumed to be globally Lipschitz continuous on $\mathbb{R}$, so that we incorporate here some ideas from [8] in order to deal with our polynomial nonlinearity.

4.1. Projection operators and discrete Laplacian. We recall that we use a conforming $\mathbb{P}_{k}(k \geq 1)$ finite element approximation $V_{h} \subset V$, based on a regular family of triangulations $\left(\mathcal{T}_{h}\right)_{h \in J}$ (cf. Section 3.1). Under these assumptions, there exists a constant $C$ independent of $h$ such that [21]

$$
\left|I_{h} v-v\right|_{H}+h\left\|I_{h} v-v\right\|_{V} \leq C h^{2}\|v\|_{H^{2}(\Omega)}, \quad \forall v \in V \cap H^{2}(\Omega),
$$

where $I_{h}: V \cap H^{2}(\Omega) \rightarrow V_{h}$ is the $P^{1}$ interpolate. That is, $I_{h} v$ is continuous on $\bar{\Omega}$, affine on every $d$-simplex of the triangulation $\mathcal{T}_{h}$, takes the sames values as $v$ on the vertices of $\mathcal{T}_{h}$, and takes the value 0 on $\bar{\Omega} \backslash \Omega_{h}$.

Remark 4.1. We stress that $I_{h}$ is the $P^{1}$ interpolate even if $k \geq 2$, since this is sufficient for our purpose. As a consequence, the error estimate with $H^{1}$ data is the same for $k=1$ and for $k \geq 2$ (Theorem 4.6). In any case, we cannot expect a much better global error estimate for $k \geq 2$, because the boundary $\Gamma_{h}$ is only a polygonal approximation of $\Gamma[21]$. In this paper, choosing $\mathbb{P}_{k}$ elements is interesting because it shows that the approach is not limited to $\mathbb{P}_{1}$ elements. 
We denote $P_{h}: L^{2} \rightarrow V_{h}$ the $L^{2}$ projection operator on $V_{h}$, defined by

$$
\left(P_{h} v, w_{h}\right)_{H}=\left(v, w_{h}\right)_{H} \quad \forall v \in H, \forall w_{h} \in V_{h},
$$

and $\Pi_{h}: V \rightarrow V_{h}$ the elliptic projection operator on $V_{h}$, defined by

$$
\left(\nabla \Pi_{h} v, \nabla w_{h}\right)_{0}=\left(\nabla v, \nabla w_{h}\right)_{0} \quad \forall v \in V, \forall w_{h} \in V_{h} .
$$

It follows from these definitions that

$$
\left|P_{h} v\right|_{H} \leq|v|_{H}, \quad \forall v \in H,
$$

and

$$
\left\|\Pi_{h} v\right\|_{V} \leq\|v\|_{V}, \quad \forall v \in V .
$$

Using standard arguments [25], we deduce from the estimates above that

$$
\left|\Pi_{h} v-v\right|_{H} \leq M_{1} h\|v\|_{V}, \quad \forall v \in V,
$$

and

$$
\left|\Pi_{h} v-v\right|_{H}+h\left\|\Pi_{h} v-v\right\|_{V} \leq M_{2} h^{2}\|v\|_{H^{2}(\Omega)}, \quad \forall v \in V \cap H^{2}(\Omega),
$$

for some constants $M_{1}$ and $M_{2}$ which are independent of $h$.

Next, we introduce the "discrete Laplacian operator" $A_{h}: V_{h} \rightarrow V_{h}$, defined by

$$
\left(A_{h} v_{h}, w_{h}\right)_{H}=\left(\nabla v_{h}, \nabla w_{h}\right)_{0} \quad \forall v_{h}, w_{h} \in V_{h} .
$$

It is clear that $A_{h}$ is a positive definite operator on $V_{h}$. We denote $G_{h}=A_{h}^{-1}$ its inverse, which is characterized by

$$
\left(\nabla G_{h} f_{h}, \nabla w_{h}\right)_{0}=\left(f_{h}, w_{h}\right)_{H}, \quad \forall f_{h}, w_{h} \in V_{h} .
$$

The following result will prove useful (cf. [8, Lemma 6.2]).

Lemma 4.2. If $\|v\|_{V} \leq R_{1}$ and $\|w\|_{V} \leq R_{1}$, then

$$
\left|G_{h}^{1 / 2} P_{h}(g(v)-g(w))\right|_{H} \leq C\left(R_{1}\right)|v-w|_{H} .
$$

Proof. For $f \in H$, on setting $w_{h}=G_{h}^{-1 / 2} \varphi_{h}=A_{h}^{1 / 2} \varphi_{h}$, we find that

$$
\left|G_{h}^{1 / 2} P_{h} f\right|_{H}=\sup _{w_{h} \in V_{h}} \frac{\left(G_{h}^{1 / 2} P_{h} f, w_{h}\right)_{H}}{\left|w_{h}\right|_{H}}=\sup _{\varphi_{h} \in V_{h}} \frac{\left|\left(f, \varphi_{h}\right)_{H}\right|}{\left\|\varphi_{h}\right\|_{V}} \leq C\|f\|_{L^{6 / 5}},
$$

since $V$ is continuously imbedded in $L^{6}(\Omega)$. Next, we use that $g(v)-g(w)=l(v-w)$ with $l=\int_{0}^{1} g^{\prime}(s v+(1-s) w) d s$, and so

$$
\|g(v)-g(w)\|_{L^{6 / 5}} \leq\|l\|_{L^{3}}\|v-w\|_{L^{2}} \leq C\left(R_{1}\right)|v-w|_{H} .
$$

The proof is complete.

4.2. Error estimates for the linear evolution operator. Let $E(t)$ denote the solution operator for the linear heat equation, i.e. $E(t) v_{0}=v(t)$ where $v$ solves

$$
\left\{\begin{array}{l}
\partial_{t} v-\Delta v=0 \quad x \in \Omega, t>0 \\
v=0 \quad(x, t) \in \partial \Omega, t>0 \\
v(x, 0)=v_{0}(x) \quad x \in \Omega
\end{array}\right.
$$

Similarly, we denote $E_{h}(t)$ the solution operator for space discrete linear problem, i.e. $E_{h}(t) v_{h}^{0}=v_{h}(t)$ where $v_{h} \in C^{1}\left(\mathbb{R}_{+}, V_{h}\right)$ solves

$$
\left\{\begin{array}{l}
\frac{d}{d t}\left(v_{h}(t), \varphi_{h}\right)_{H}+\left(\nabla v_{h}(t), \nabla \varphi_{h}\right)_{0}=0 \quad \text { for } t \geq 0, \forall \varphi_{h} \in V_{h}, \\
v_{h}(0)=v_{h}^{0}
\end{array}\right.
$$


If we use the discrete Laplacian $A_{h}$ (cf. (4.5)), this can be written

$$
\begin{gathered}
\frac{d v_{h}(t)}{d t}+A_{h} v_{h}(t)=0, \quad t \geq 0, \\
v_{h}(0)=v_{h}^{0} .
\end{gathered}
$$

On choosing $\varphi_{h}=v_{h}(t)$ in (4.7), we see that $t \mapsto\left|v_{h}(t)\right|_{H}^{2}$ is nonincreasing. Similarly, $t \mapsto|v(t)|_{H}^{2}$ is nonincreasing. In particular, for every $t \geq 0, w \in H$ and $w_{h} \in V_{h}$, we have

$$
|E(t) w|_{H} \leq|w|_{H} \quad \text { and } \quad\left|E_{h}(t) w_{h}\right|_{H} \leq\left|w_{h}\right|_{H} .
$$

The following error estimates with nonsmooth data are standard (cf. [25, Theorem 3.1 and Theorem 3.2]).

Proposition 4.3. There exists a constant $C$ such that for all $w \in V$ and for all $h \in J$,

$$
\left|E_{h}(t) P_{h} w-E(t) w\right|_{H} \leq C h\|w\|_{V}, \quad \text { for } t \geq 0 .
$$

Proposition 4.4. There exists a constant $C$ such that for all $w \in H$ and for all $h \in J$,

$$
\left|E_{h}(t) P_{h} w-E(t) w\right|_{H} \leq C h^{2} t^{-1}|w|_{H}, \quad \text { for } t>0 .
$$

We will use the following $H-V$ smoothing property.

Lemma 4.5. For all $w_{h} \in V_{h}$, we have

$$
\left|A_{h}^{1 / 2} E_{h}(t) w_{h}\right|_{H} \leq t^{-1 / 2}\left|w_{h}\right|_{H}, \quad \forall t>0 .
$$

Proof. Let $v_{h}=E_{h}(t) w_{h}$. We choose $\varphi_{h}=\frac{d v_{h}(t)}{d t}$ in (4.7), we multiply the resulting equation by $2 t$ and we add $\left\|v_{h}(t)\right\|_{V}^{2}$. We obtain

$$
\frac{d}{d t}\left(t\left\|v_{h}(t)\right\|_{V}^{2}\right) \leq\left\|v_{h}(t)\right\|_{V}^{2}
$$

Integrating on $[0, t]$ yields

$$
t\left\|v_{h}(t)\right\|_{V}^{2} \leq \int_{0}^{t}\left\|v_{h}(s)\right\|_{V}^{2} d s .
$$

Next, we choose $\varphi_{h}=v_{h}(t)$ in (4.7), and we integrate on $[0, t]$. We find

$$
\frac{1}{2}\left|v_{h}(t)\right|_{H}^{2}+\int_{0}^{t}\left\|v_{h}(s)\right\|_{V}^{2} d s \leq \frac{1}{2}\left|w_{h}\right|_{H}^{2} .
$$

Thus, for all $t>0$, we have $t\left\|v_{h}(t)\right\|_{V}^{2} \leq\left|w_{h}\right|_{H}^{2}$, that is (4.9).

4.3. Error estimates for the semilinear evolution problem. With the notation above, the space semidiscrete semilinear problem (3.2)-(3.3) reads

$$
\begin{aligned}
\frac{d u_{h}(t)}{d t}+A_{h} u_{h}(t)+P_{h} g\left(u_{h}(t)\right) & =0, \quad \forall t \geq 0, \\
u_{h}(0) & =u_{h}^{0} .
\end{aligned}
$$

We recall that $u$ solves the semilinear problem (2.1)-(2.2). We obtain the following error estimate, which is not necessarily optimal (compare with $[9,15])$, but which will prove sufficient for our purpose. 
Theorem 4.6. For all $R_{1}>0$ and for all $T>0$, there exists a constant $C_{3}\left(R_{1}, T\right)$ independent of $h$ such that, if $\left\|u_{0}\right\|_{V} \leq R_{1}$ and $u_{h}^{0}=\Pi_{h}\left(u_{0}\right)$, then

$$
\sup _{t \in[0, T]}\left|u_{h}(t)-u(t)\right|_{H} \leq C_{3}\left(R_{1}, T\right) h .
$$

Proof. Using Lemma 2.5 and Lemma 3.3, we find that

$$
\|u(t)\|_{V} \leq C_{1}\left(R_{1}\right) \quad \text { and } \quad\left\|u_{h}(t)\right\|_{V} \leq C_{1}\left(R_{1}\right), \quad \forall t \geq 0 .
$$

As a consequence, $g(u)$ and $g\left(u_{h}\right)$ are bounded in $L^{\infty}\left(\mathbb{R}_{+} ; H\right)$, uniformly with respect to $h$. By Duhamel's principle, $u$ satisfies

$$
u(t)=E(t) u_{0}-\int_{0}^{t} E(t-s) g(u(s)) d s
$$

and $u_{h}$ satisfies

$$
u_{h}(t)=E_{h}(t) u_{h}^{0}-\int_{0}^{t} E_{h}(t-s) P_{h} g\left(u_{h}(s)\right) d s .
$$

We denote $e_{h}(t)=u_{h}(t)-u(t)$.

Using the triangle inequality, estimate (4.8) and Proposition 4.3, we have, for the linear terms,

$$
\begin{aligned}
\left|E_{h}(t) u_{h}^{0}-E(t) u_{0}\right|_{H} & \leq\left|E_{h}(t) \Pi_{h} u_{0}-E_{h}(t) P_{h} u_{0}\right|_{H}+\left|E_{h}(t) P_{h} u_{0}-E(t) u_{0}\right|_{H} \\
& \leq\left|\Pi_{h} u_{0}-P_{h} u_{0}\right|_{H}+C h\left\|u_{0}\right\|_{V}
\end{aligned}
$$

Since $\Pi_{h} u_{0}=P_{h} \Pi_{h} u_{0}$, we have, using (4.2) and (4.4),

$$
\left|\Pi_{h} u_{0}-P_{h} u_{0}\right|_{H}=\left|P_{h}\left[\Pi_{h} u_{0}-u_{0}\right]\right|_{H} \leq M_{1} h\left\|u_{0}\right\|_{V},
$$

and we find that

$$
\left|E_{h}(t) u_{h}^{0}-E(t) u_{0}\right|_{H} \leq C\left(R_{1}\right) h, \quad \forall t \geq 0,
$$

where here and below, $C\left(R_{1}\right)$ is a generic constant depending on $R_{1}$, but independent of $h$ and $T$.

For $0 \leq t \leq h^{2}$, by using the bound on $g(u), g\left(u_{h}\right)$ and (4.8), we have

$$
\begin{aligned}
\left|e_{h}(t)\right|_{H} & \leq\left|E_{h}(t) u_{h}^{0}-E(t) u_{0}\right|_{H}+C\left(R_{1}\right) t \\
& \leq C\left(R_{1}\right) h .
\end{aligned}
$$

In the second inequality, we used (4.10) and $t \leq h^{2} \leq h_{\max } h$.

For $t \geq h^{2}$, we write

$$
\begin{aligned}
e_{h}(t)= & \left(E_{h}(t) P_{h} u_{0}-E(t) u_{0}\right)-\int_{0}^{t} E_{h}(t-s) P_{h}\left[g\left(u_{h}(s)\right)-g(u(s))\right] d s \\
& -\int_{0}^{t} E_{h}(t-s) P_{h} g(u(s))-E(t-s) g(u(s)) d s .
\end{aligned}
$$

In the right-hand side above, for the first term, we use (4.10) again. We cut the first integral into an integral over $\left[0, h^{2}\right]$ (for which we use the same $L^{2}$-bounds as previously), and an integral over $\left[h^{2}, t\right]$. We cut the second integral into an integral 
over $\left[0, t-h^{2}\right]$ (for which we use Proposition 4.4) and an integral over $\left[t-h^{2}, t\right]$ (for which we use the $L^{2}$-bounds). This yields

$$
\begin{aligned}
\left|e_{h}(t)\right|_{H} \leq & C h\left\|u_{0}\right\|_{V}+\int_{0}^{h^{2}} C\left(R_{1}\right) d s \\
& +\int_{h^{2}}^{t}\left|A_{h}^{1 / 2} E_{h}(t-s) G_{h}^{1 / 2} P_{h}\left[g\left(u_{h}(s)\right)-g(u(s))\right]\right|_{H} d s \\
& +\int_{0}^{t-h^{2}} C h^{2}(t-s)^{-1}|g(s)|_{H} d s+\int_{t-h^{2}}^{t} C\left(R_{1}\right) d s .
\end{aligned}
$$

For the integral over $\left[h^{2}, t\right]$, we use successively Lemma 4.5 and Lemma 4.2. This yields

$\left|e_{h}(t)\right|_{H} \leq C\left(R_{1}\right) h+C\left(R_{1}\right) h^{2}+C\left(R_{1}\right) h^{2} \ln \left(t / h^{2}\right)+C\left(R_{1}\right) \int_{h^{2}}^{t}(t-s)^{-1 / 2}\left|e_{h}(s)\right|_{H} d s$.

Thus, for $h^{2} \leq t \leq T$, we have

$$
\left|e_{h}(t)\right|_{H} \leq C^{\prime}\left(R_{1}, T\right) h+C\left(R_{1}\right) \int_{h^{2}}^{t}(t-s)^{-1 / 2}\left|e_{h}(s)\right|_{H} d s,
$$

where $C^{\prime}\left(R_{1}, T\right)$ is independent of $h$. Now, we may apply a standard generalization of Gronwall's lemma (see [8, Lemma 6.3]), and we find that for some constant $C^{\prime \prime}\left(R_{1}, T\right)$, we have

$$
\left|e_{h}(t)\right|_{H} \leq C^{\prime \prime}\left(R_{1}, T\right) h, \quad h^{2} \leq t \leq T .
$$

This concludes the proof.

\section{A Perturbation Result And a first CONSEQuence}

5.1. Global attractor and exponential attractor. We recall some standard definitions (see e.g. $[18,24]$ ). Throughout Section $5.1, \mathcal{K}$ denotes a closed subset of the Hilbert space $H$. A continuous-in-time semigroup $\left\{S(t), t \in \mathbb{R}_{+}\right\}$on $\mathcal{K}$ is a family of (nonlinear) operators such that $S(t)$ is a continuous operator from $\mathcal{K}$ into itself, for all $t \geq 0$, with $S(0)=I d$ (identity in $\mathcal{K}$ ) and

$$
S(t+s)=S(t) \circ S(s), \quad \forall s, t \geq 0 .
$$

A discrete-in-time semigroup $\{S(t), t \in \mathbb{N}\}$ on $\mathcal{K}$ is a family of (nonlinear) operators which satisfy these properties with $\mathbb{R}_{+}$replaced by $\mathbb{N}$. A discrete-in-time semigroup is usually denoted $\left\{S^{n}, n \in \mathbb{N}\right\}$, where $S(=S(1)$ ) is a continuous (nonlinear) operator from $\mathcal{K}$ into itself. The term "dynamical system" will sometimes be used instead of "semigroup".

Definition 5.1 (Global attractor). Let $\{S(t), t \geq 0\}$ be a continuous or discrete semigroup on $\mathcal{K}$. A set $\mathcal{A} \subset \mathcal{K}$ is called the global attractor of the dynamical system if the following three conditions are satisfied:

(1) $\mathcal{A}$ is compact in $H$;

(2) $\mathcal{A}$ is invariant, i.e. $S(t) \mathcal{A}=\mathcal{A}$, for all $t \geq 0$;

(3) $\mathcal{A}$ attracts all bounded sets in $\mathcal{K}$, i.e., for every bounded set $B$ in $\mathcal{K}$,

$$
\lim _{t \rightarrow+\infty} \operatorname{dist}_{H}(S(t) B, \mathcal{A})=0
$$


Here, dist $_{H}$ denotes the non-symmetric Hausdorff semidistance in $H$ between two subsets, which is defined as

$$
\operatorname{dist}_{H}(A, B)=\sup _{a \in A} \inf _{b \in B}|a-b|_{H} .
$$

It is easy to see, thanks to the invariance and the attracting property, that the global attractor, when it exists, is unique [24].

Let $A \subset H$ be a (relatively compact) subset of $H$. For $\varepsilon>0$, we denote $N_{\varepsilon}(A, H)$ the minimum number of balls of $H$ of radius $\varepsilon>0$ which are necessary to cover $A$. The fractal dimension of $A$ (see e.g. $[5,24])$ is the number

$$
\operatorname{dim}_{F}(A, H)=\limsup _{\varepsilon \rightarrow 0} \frac{\log _{2}\left(N_{\varepsilon}(A, H)\right)}{\log _{2}(1 / \varepsilon)} \in[0,+\infty]
$$

Definition 5.2 (Exponential attractor). Let $\{S(t), t \geq 0\}$ be a continuous or discrete semigroup on $\mathcal{K}$. A set $\mathcal{M} \subset \mathcal{K}$ is an exponential attractor of the dynamical system if the following three conditions are satisfied:

(1) $\mathcal{M}$ is compact in $H$ and has finite fractal dimension;

(2) $\mathcal{M}$ is positively invariant, i.e. $S(t) \mathcal{M} \subset \mathcal{M}$, for all $t \geq 0$;

(3) $\mathcal{M}$ attracts exponentially the bounded subsets of $\mathcal{K}$ in the following sense:

$\forall B \subset \mathcal{K}$ bounded, $\quad \operatorname{dist}_{H}(S(t) B, \mathcal{M}) \leq \mathcal{Q}\left(\left\|B_{h}\right\|_{H}\right) e^{-\alpha t}, \quad t \geq 0$,

where the positive constant $\alpha$ and the monotonic function $\mathcal{Q}$ are independent of $B$. Here, $\|B\|_{H}=\sup _{b \in B}|b|_{H}$.

The exponential attractor, if it exists, contains the global attractor (actually, the existence of an exponential attractor yields the existence of the global attractor, see $[2,4])$. We also note that if $\mathcal{K}$ is bounded, then in the definition above, we may obviously replace (3) by:

(3bis) $\mathcal{M}$ attracts $\mathcal{K}$ exponentially, i.e.

$$
\operatorname{dist}_{H}(S(t) \mathcal{K}, \mathcal{M}) \leq C e^{-\alpha t}, \quad t \geq 0
$$

for some positive constants $C$ and $\alpha$.

5.2. Construction of a robust family of exponential attractors. We introduce the following absorbing sets,

$$
\mathcal{B}_{0}=\left\{v \in V:\|v\| \leq \mathcal{R}_{1}\right\} \quad \text { and } \quad \mathcal{B}_{h}=\left\{v_{h} \in V_{h}:\left\|v_{h}\right\| \leq \mathcal{R}_{1}\right\},
$$

for all $h \in J$. We note that $\Pi_{h}\left(\mathcal{B}_{0}\right)=\mathcal{B}_{h}$, and this will make our construction of exponential attractors much easier. Indeed, since $\mathcal{B}_{h} \subset \mathcal{B}_{0}$ and $\Pi_{h}\left(\mathcal{B}_{h}\right)=\mathcal{B}_{h}$, it is clear that $\mathcal{B}_{h} \subset \Pi_{h}\left(\mathcal{B}_{0}\right)$. But we also have $\Pi_{h}\left(\mathcal{B}_{0}\right) \subset \mathcal{B}_{h}$, because $\left\|\Pi_{h}(v)\right\|_{V} \leq\|v\|_{V}$ for all $v \in V$.

Thanks to Proposition 2.4 and Proposition 3.2, for $T>0$ large enough independent of $h$, we have

$$
S_{0}(T)\left(\mathcal{B}_{0}\right) \subset \mathcal{B}_{0} \quad \text { and } \quad \forall h \in J, S_{h}(T)\left(\mathcal{B}_{h}\right) \subset \mathcal{B}_{h} ;
$$

we may choose, for instance, $T=\mathcal{T}_{0}\left(c_{0} \mathcal{R}_{1}\right)+r$. As a shortcut, we denote $L_{0}=S_{0}(T)$ and, for every $h \in J, L_{h}=S_{h}(T)$. We will also write $\bar{J}=\{0\} \cup J$.

We first collect some useful facts.

Proposition 5.3. The following properties hold: 
(P1) Smoothing property, uniform in $h:$ For all $h \in \bar{J}$ and for all $v_{h}$, $w_{h} \in \mathcal{B}_{h}$,

$$
\left\|L_{h} v_{h}-L_{h} w_{h}\right\|_{V} \leq c_{1}\left|v_{h}-w_{h}\right|_{H}
$$

where the constant $c_{1}$ is independent of $h$.

(P2) Estimate between the trajectories: For all $h \in \bar{J}$, for all $v_{h} \in \mathcal{B}_{h}$ and for all $n \in \mathbb{N}$,

$$
\left|L_{h}^{n} v_{h}-L_{0}^{n} v_{h}\right|_{H} \leq c_{2} c_{3}^{n} h,
$$

where the constants $c_{2}, c_{3}$ are independent of $h$.

(P3) Lipschitz continuity of $L_{0}$ : There exists a constant $c_{4}>1$ such that for all $v, w \in H$ and for all $n \in \mathbb{N}$,

$$
\left|L_{0}^{n} v-L_{0}^{n} w\right|_{H} \leq c_{4}^{n}|v-w|_{H} .
$$

(P4) Estimate for the projection operator: For all $h \in J$, for all $v \in V$,

$$
\left|v-\Pi_{h} v\right|_{H} \leq M_{1} h\|v\|_{V} .
$$

Proof. We prove property (P2) (the three other properties are a direct consequence of estimates established in previous sections).

Let $h \in J$. For $v_{h} \in \mathcal{B}_{h}$, we apply Theorem 4.6 with $u_{0}=v_{h}$ and $u_{h}^{0}=\Pi_{h}\left(v_{h}\right)=$ $v_{h}$. This yields

$$
\left|L_{h} v_{h}-L_{0} v_{h}\right|_{H} \leq C_{3}\left(\mathcal{R}_{1}, T\right) h .
$$

Now, we assume by induction that for some $n \geq 1$, we have

$$
\left|L_{h}^{n} v_{h}-L_{0}^{n} v_{h}\right|_{H} \leq\left(\sum_{j=0}^{n-1} c_{4}^{j}\right) C_{3}\left(\mathcal{R}_{1}, T\right) h,
$$

for all $v_{h} \in \mathcal{B}_{h}$. Then, for $v_{h} \in \mathcal{B}_{h}$, we can write

$$
\begin{aligned}
\left|L_{h}^{n+1} v_{h}-L_{0}^{n+1} v_{h}\right|_{H} & \leq\left|L_{h}^{n}\left(L_{h} v_{h}\right)-L_{0}^{n}\left(L_{h} v_{h}\right)\right|_{H}+\left|L_{0}^{n}\left(L_{h} v_{h}\right)-L_{0}^{n}\left(L_{0} v_{h}\right)\right|_{H} \\
& \leq\left(\sum_{j=0}^{n-1} c_{4}^{j}\right) C_{3}\left(\mathcal{R}_{1}, T\right) h+c_{4}^{n} C_{3}\left(\mathcal{R}_{1}, T\right) h,
\end{aligned}
$$

where we used that $L_{h} v_{h} \in \mathcal{B}_{h}$, property (P3) and (5.3). By induction, estimate (5.4) holds for all $n \geq 1$. This proves (P2) with $c_{2}=C_{3}\left(\mathcal{R}_{1}, T\right) /\left(c_{4}-1\right)$ and $c_{3}=c_{4}$.

Our final convergence result is based on the following essential result, whose proof is adapted from $[7,12]$.

Lemma 5.4. For every $h \in \bar{J}$, there exists an exponential attractor $\mathcal{M}_{h}^{d}$ for (the iterates of) the map $L_{h}: \mathcal{B}_{h} \rightarrow \mathcal{B}_{h}$ such that:

(1) the fractal dimension of $\mathcal{M}_{h}^{d}$ in $H$ is bounded, uniformly with respect to $h \in \bar{J}$,

$$
\operatorname{dim}_{F}\left(\mathcal{M}_{h}^{d}, H\right) \leq c_{5},
$$

where $c_{5}$ is independent of $h$;

(2) $\mathcal{M}_{h}^{d}$ attracts $\mathcal{B}_{h}$ uniformly with respect to $h \in \bar{J}$, i.e.

$$
\operatorname{dist}_{H}\left(L_{h}^{n} \mathcal{B}_{h}, \mathcal{M}_{h}^{d}\right) \leq c_{6} 2^{-n}, \quad n \in \mathbb{N},
$$

where $c_{6}$ is independent of $h$; 
(3) the family $\left\{\mathcal{M}_{h}^{d}, h \in \bar{J}\right\}$ is continuous at 0 ,

$$
\operatorname{dist}_{\text {sym }}\left(\mathcal{M}_{h}^{d}, \mathcal{M}_{0}^{d}\right) \leq c_{7} h^{\kappa},
$$

where $c_{7}$ and $\kappa \in(0,1)$ are independent of $h$ and dist sym $_{\text {denotes the sym- }}$ metric Hausdorff distance between sets, defined by

$$
\operatorname{dist}_{\text {sym }}(A, B):=\max \left\{\operatorname{dist}_{H}(A, B), \operatorname{dist}_{H}(B, A)\right\} .
$$

Proof. We first construct an exponential attractor $\mathcal{M}_{0}^{d}$ for $L_{0}$. To this end (see also $[6,7]$ ), we construct $R / 2^{n}$ coverings of the sets $L_{0}^{n} \mathcal{B}_{0}$ by the following inductive procedure:

1. Since the set $\mathcal{B}_{0}$ is bounded in $H$, there exists a ball $B\left(v_{0}, R, H\right)$ of radius $R$ centered at $v_{0} \in \mathcal{B}_{0}$ for the $H$-norm such that $\mathcal{B}_{0} \subset B\left(v_{0}, R, H\right)$. We set $W_{0}^{0}=E_{0}^{0}:=$ $\left\{v_{0}\right\}$. Thus, we have constructed the initial $R$-covering of the set $\mathcal{B}_{0}$.

2 . We now assume that the $R / 2^{n}$-covering of the set $L_{0}^{n} \mathcal{B}_{0}$ by the balls centered at the points of the set $W_{0}^{n} \subset L_{0}^{n} \mathcal{B}_{0}$ is already constructed. Then, according to (5.2), the system of $c_{1} R / 2^{n}$-balls for the $V$-metric centered at the points $\left(v_{0, j}\right)_{j}$ of $L_{0} W_{0}^{n}$ covers the set $L_{0}^{n+1} \mathcal{B}_{0}$. By Rellich's theorem, the imbedding $V \subset H$ is compact [14]. Thus, every $V$-ball is compact for the $H$-metric, and we can cover each $c_{1} R / 2^{n}$-ball for $V$-metric from the above covering by a finite number $P$ of $R /\left(4 \cdot 2^{n}\right)$-balls for the $H$-metric. Moreover, the number $P$ can be computed as follows:

$$
\begin{aligned}
P & =N_{R / 2^{n+2}}\left(B\left(v_{0, j}, c_{1} R / 2^{n}, V\right), H\right) \\
& =N_{R / 2^{n+2}}\left(B\left(0, c_{1} R / 2^{n}, V\right), H\right)=N_{1 / 4 c_{1}}(B(0,1, V), H) .
\end{aligned}
$$

Here, we denote $N_{\varepsilon}(K, H)$ the minimal number of $\varepsilon$-balls for the $H$-norm which are necessary to cover the (relatively) compact set $K \subset H$. The relation (5.5) shows that $P$ is independent of $n$. Thus, we have constructed a $R / 2^{n+2}$-covering $\mathcal{U}_{0}^{n+1}$ of the set $L_{0}^{n+1} \mathcal{B}_{0}$ by a number of balls that is not greater than

$$
\# \mathcal{U}_{0}^{n+1} \leq P \# W_{0}^{n} .
$$

By increasing the radiuses of the balls by a factor two if necessary, we can construct a $R / 2^{n+1}$-covering with centers belonging to $L_{0}^{n+1} \mathcal{B}$. Thus, having the set $W_{0}^{n}$, we have constructed the set $W_{0}^{n+1}$ such that

$$
\# W_{0}^{n+1} \leq P \# W_{0}^{n}
$$

and

$$
\operatorname{dist}_{H}\left(L_{0}^{n+1} \mathcal{B}_{0}, W_{0}^{n+1}\right) \leq R / 2^{n+1}, \quad W_{0}^{n+1} \subset L_{0}^{n+1} \mathcal{B}_{0} .
$$

Finally, we set $E_{0}^{n+1}:=L_{0} E_{0}^{n} \cup W_{0}^{n+1}$. Then, due to the induction procedure, the sets $E_{0}^{n}, n \in \mathbb{N}$, enjoy the following properties:

$$
\left\{\begin{array}{l}
\text { 1. } E_{0}^{n} \subset L_{0}^{n} \mathcal{B}_{0}, \\
\text { 2. } L_{0} E_{0}^{n} \subset E_{0}^{n+1}, \\
\text { 3. } \# E_{0}^{n} \leq P^{n+1}, \\
\text { 4. } \text { dist }_{H}\left(L_{0}^{n} \mathcal{B}_{0}, E_{0}^{n}\right) \leq R / 2^{n} .
\end{array}\right.
$$

We now define the exponential attractor $\mathcal{M}_{0}^{d}$ as follows:

$$
\mathcal{M}_{0}^{\prime}=\bigcup_{n=1}^{\infty} E_{0}^{n}, \quad \mathcal{M}_{0}^{d}=\left[\mathcal{M}_{0}^{\prime}\right]_{H},
$$


where $[\cdot]_{H}$ denotes the closure in $H$. It is easy to verify, using (5.6), that the set $\mathcal{M}_{0}^{d}$ is indeed an exponential attractor for the map $L_{0}: \mathcal{B}_{0} \rightarrow \mathcal{B}_{0}$.

Let us now construct the exponential attractors $\mathcal{M}_{h}$ for the maps $L_{h}$. To this end, we will essentially use the sets $E_{0}^{n}$ constructed above. Since $E_{0}^{n} \subset L_{0}^{n} \mathcal{B}_{0}$, there exist sets $\tilde{E}_{0}^{n} \subset \mathcal{B}_{0}$ such that

$$
\# \tilde{E}_{0}^{n}=\# E_{0}^{n}, \quad L_{0}^{n} \tilde{E}_{0}^{n}=E_{0}^{n} .
$$

For $h \in J$, we define the sets

$$
\tilde{E}_{h}^{n}=\Pi_{h} \tilde{E}_{0}^{n} \quad \text { and } \quad \hat{E}_{h}^{n}=L_{h}^{n} \tilde{E}_{h}^{n} .
$$

Next, we estimate $\operatorname{dist}_{H}\left(L_{h}^{n} \mathcal{B}_{h}, \hat{E}_{h}^{n}\right)$. Let $v_{h} \in \mathcal{B}_{h}$ and $\tilde{v}_{0} \in \tilde{E}_{0}^{n}$. We set $\hat{v}_{h}^{n}=$ $L_{h}^{n} \Pi_{h} \tilde{v}_{0} \in \hat{E}_{h}^{n}$. We have

$$
\begin{aligned}
\left|L_{h}^{n} v_{h}-\hat{v}_{h}^{n}\right|_{H} \leq & \left|L_{h}^{n} v_{h}-L_{0}^{n} v_{h}\right|_{H}+\left|L_{0}^{n} v_{h}-L_{0}^{n} \tilde{v}_{0}\right|_{H} \\
& +\left|L_{0}^{n} \tilde{v}_{0}-L_{0}^{n} \Pi_{h} \tilde{v}_{0}\right|_{H}+\left|L_{0}^{n} \Pi_{h} \tilde{v}_{0}-L_{h}^{n} \Pi_{h} \tilde{v}_{0}\right|_{H} \\
\leq & 2 c_{2} c_{3}^{n} h+\left|L_{0}^{n} v_{h}-v_{0, j}\right|_{H}+c_{4}^{n} M_{1} \mathcal{R}_{1} h
\end{aligned}
$$

where $v_{0, j}=L_{0}^{n} \tilde{v}_{0}$ is in the set $E_{0}^{n}$. Since $v_{h} \in \mathcal{B}_{0}$, we may choose $v_{0, j}$ such that $\left|L_{0}^{n} v_{h}-v_{0, j}\right|_{H} \leq R / 2^{n}$, and we have

$$
\left|L_{h}^{n} v_{h}-\hat{v}_{h}^{n}\right|_{H} \leq 2 c_{2} c_{3}^{n} h+c_{4}^{n} M_{1} \mathcal{R}_{1} h+R / 2^{n} \leq c_{8} c_{9}^{n} h+R / 2^{n},
$$

with $c_{9}=\max \left\{c_{3}, c_{4}\right\}>1$ and $c_{8}=2 c_{2}+M_{1} \mathcal{R}_{1}$. We define $N=N(h) \geq 0$ as the real number such that $c_{8} c_{9}^{N} h=R / 2^{N}$, i.e.

$$
N(h)=\frac{\ln \left(R /\left(c_{8} h\right)\right)}{\ln \left(2 c_{9}\right)}
$$

(we may increase $R$ if necessary so that $R \geq c_{8} h_{\max }$ ). For $0 \leq n \leq N(h)$, we have

$$
\operatorname{dist}_{H}\left(L_{h}^{n} \mathcal{B}_{h}, \hat{E}_{h}^{n}\right) \leq R / 2^{n-1} .
$$

Now, we note that for all $\tilde{v}_{0} \in \tilde{E}_{0}^{n}$, we have

$$
\begin{aligned}
\left|L_{h}^{n} \Pi_{h} \tilde{v}_{0}-L_{0}^{n} \tilde{v}_{0}\right|_{H} & \leq\left|L_{h}^{n} \Pi_{h} \tilde{v}_{0}-L_{0}^{n} \Pi_{h} \tilde{v}_{0}\right|_{H}+\left|L_{0}^{n} \Pi_{h} \tilde{v}_{0}-L_{0}^{n} \tilde{v}_{0}\right|_{H} \\
& \leq c_{2} c_{3}^{n} h+c_{4}^{n} M_{1} \mathcal{R}_{1} h \\
& \leq c_{8} c_{9}^{n} h,
\end{aligned}
$$

and so

$$
\operatorname{dist}_{\text {sym }}\left(\hat{E}_{h}^{n}, E_{0}^{n}\right) \leq c_{8} c_{9}^{n} h .
$$

Using the definition (5.7) of $N(h)$, we see that for all $0 \leq n \leq N(h)$, we have $c_{8} c_{9}^{n} h \leq C_{4} h^{\kappa}$ where $\kappa=\ln 2 /\left(\ln 2+\ln c_{9}\right) \in(0,1)$ and $C_{4}=C\left(R, c_{8}, c_{9}\right)$. Thus, for $0 \leq n \leq N(h)$, we have

$$
\operatorname{dist}_{\text {sym }}\left(\hat{E}_{h}^{n}, E_{0}^{n}\right) \leq C_{4} h^{\kappa} .
$$

Finally, we define the sets $E_{h}^{n}$ as follows:

(1) If $0 \leq n \leq N(h)$, then we set $E_{h}^{n}=\hat{E}_{h}^{n}$;

(2) If $n>N(h)$, we forget the sets $E_{h}^{n}$ and we construct the sets $E_{h}^{n}$ in $\mathcal{B}_{h}$ by an inductive procedure, using estimate (5.2); we proceed as in the construction of the sets $E_{0}^{n}$, but starting with the set $\hat{E}_{h}^{\lfloor N(h)\rfloor}$ (here $\lfloor\cdot\rfloor$ is the floor integer function). 
The sets $E_{h}^{n}$ clearly satisfy

$$
\left\{\begin{array}{l}
\text { 1. } E_{h}^{n} \subset L_{h}^{n} \mathcal{B}_{h}, \\
\text { 2. } L_{h} E_{h}^{n} \subset E_{h}^{n+1} \\
\text { 3. \# } E_{h}^{n} \leq P^{n+2} \\
\text { 4. } \operatorname{dist}_{H}\left(L_{h}^{n} \mathcal{B}_{h}, E_{h}^{n}\right) \leq R / 2^{n-1} .
\end{array}\right.
$$

It follows from (5.9) that the set

$$
\mathcal{M}_{h}^{d}=\left[\mathcal{M}_{h}^{\prime}\right]_{H} \quad \text { where } \quad \mathcal{M}_{h}^{\prime}=\bigcup_{n=1}^{\infty} E_{h}^{n}
$$

is an exponential attractor for the map $L_{h}: \mathcal{B}_{h} \rightarrow \mathcal{B}_{h}$, whose fractal dimension is bounded by the (explicit) constant $\log _{2}(P)$, which is independent of $h$ (see $[6,7]$ ).

Let us show that

$$
\operatorname{dist}_{H}\left(\mathcal{M}_{h}^{\prime}, \mathcal{M}_{0}^{d}\right) \leq C h^{\kappa} .
$$

Let $v_{h} \in \mathcal{M}_{h}^{\prime}$. Then $v_{h} \in E_{h}^{n}$ for some $n \geq 1$. If $1 \leq n \leq N(h)$, then (5.10) is a consequence of (5.8). Assume that $n>N(h)$. Then $v_{h} \in L_{h}^{n} \mathcal{B}_{h} \subset L_{h}^{\lfloor N(h)\rfloor} \mathcal{B}_{h}$, so there exists $\tilde{v}_{h} \in \mathcal{B}_{h}$ such that $v_{h}=L_{h}^{\lfloor N(h)\rfloor} \tilde{v}_{h}$. We consider now $v_{0}^{\star}=L_{0}^{\lfloor N(h)\rfloor} \tilde{v}_{h}$. On one hand, we have

$$
\left|v_{h}-v_{0}^{\star}\right|_{H} \leq c_{2} c_{3}^{\lfloor N(h)\rfloor} h \leq C_{4} h^{\kappa},
$$

and on the other hand, we have

$$
\operatorname{dist}_{H}\left(v_{0}^{\star}, \mathcal{M}_{0}^{d}\right)=\operatorname{dist}_{H}\left(L_{0}^{\lfloor N(h)\rfloor} \tilde{v}_{h}, \mathcal{M}_{0}^{d}\right) \leq R / 2^{\lfloor N(h)\rfloor} \leq C_{4}^{\prime} h^{\kappa} .
$$

Thus, $\operatorname{dist}_{H}\left(v_{h}, \mathcal{M}_{0}\right) \leq\left(C_{4}+C_{4}^{\prime}\right) h^{\kappa}$. Since $v_{h}$ is arbitrary, this proves (5.10).

The converse estimate,

$$
\operatorname{dist}_{H}\left(\mathcal{M}_{0}^{\prime}, \mathcal{M}_{h}^{d}\right) \leq C h^{\kappa},
$$

can be proved in a similar way. Indeed, let $v_{0} \in \mathcal{M}_{0}^{\prime}$, so that $v_{0} \in E_{0}^{n}$ for some $n \geq 1$. If $1 \leq n \leq N(h)$, then (5.8) proves (5.11). Assume that $n>N(h)$. Then $v_{0} \in L_{0}^{n} \mathcal{B}_{0} \subset L_{0}^{\lfloor N(h)\rfloor} \mathcal{B}_{0}$, so there exists $\tilde{v}_{0} \in \mathcal{B}_{0}$ such that $v_{0}=L_{0}^{\lfloor N(h)\rfloor} \tilde{v}_{0}$. We consider $v_{h}^{\star}=L_{h}^{\lfloor N(h)\rfloor} \Pi_{h} \tilde{v}_{0}$. On one hand, we have

$$
\begin{aligned}
\left|v_{0}-v_{h}^{\star}\right|_{H} & =\left|L_{0}^{\lfloor N(h)\rfloor} \tilde{v}_{0}-L_{h}^{\lfloor N(h)\rfloor} \Pi_{h} \tilde{v}_{0}\right|_{H} \\
& \leq\left|L_{0}^{\lfloor N(h)\rfloor} \tilde{v}_{0}-L_{0}^{\lfloor N(h)\rfloor} \Pi_{h} \tilde{v}_{0}\right|_{H}+\left|L_{0}^{\lfloor N(h)\rfloor} \Pi_{h} \tilde{v}_{0}-L_{h}^{\lfloor N(h)\rfloor} \Pi_{h} \tilde{v}_{0}\right|_{H} \\
& \leq c_{4}^{\lfloor N(h)\rfloor} M_{1} \mathcal{R}_{1}+c_{2} c_{3}^{\lfloor N(h)\rfloor} h \\
& \leq C_{4} h^{\kappa} .
\end{aligned}
$$

On the other hand, we have

$$
\operatorname{dist}_{H}\left(v_{h}^{\star}, \mathcal{M}_{h}^{d}\right)=\operatorname{dist}_{H}\left(L_{h}^{\lfloor N(h)\rfloor} \Pi_{h} \tilde{v}_{0}, \mathcal{M}_{h}^{d}\right) \leq R / 2^{\lfloor N(h)\rfloor} \leq C_{4}^{\prime} h^{\kappa} .
$$

This proves (5.11). From (5.10) and (5.11), we deduce that dist ${ }_{\text {sym }}\left(\mathcal{M}_{h}^{d}, \mathcal{M}_{0}^{d}\right) \leq C h^{\kappa}$, and the proof is complete. 
5.3. Convergence of global attractors. A first consequence of our construction is the following result.

Theorem 5.5. For every $h \in \bar{J}$, the semigroup $\left\{S_{h}(t), t \geq 0\right\}$ on $V_{h}$ (on $H$ if $h=0)$ possesses a global attractor $\mathcal{A}_{h}$ which is compact and connected in $H$ and bounded in $V$, uniformly in $h$. Moreover, $\operatorname{dist}_{H}\left(\mathcal{A}_{h}, \mathcal{A}_{0}\right) \rightarrow 0$ as $h \rightarrow 0$, and the fractal dimension of $\mathcal{A}_{h}$ is bounded by a constant independent of $h$.

Proof. The existence of a connected global attractor $\mathcal{A}_{h}$ for the continuous semigroup $\left\{S_{h}(t), t \geq 0\right\}$ is a consequence of Theorem 1.1 in [24] and of the estimates from Sections 2.3 and 3.3. Upper semicontinuity of $\mathcal{A}_{h}$ as $h \rightarrow 0$ is a consequence of the error estimate (Theorem 4.6) and of Theorem 4 in [27]. Now, we note that $\mathcal{A}_{h}$ is also a global attractor for the iterates of the operator $L_{h}=S_{h}(T): \mathcal{B}_{h} \rightarrow \mathcal{B}_{h}$ defined by (5.1). Thus, $\mathcal{A}_{h} \subset \mathcal{M}_{h}^{d}$, and so $\operatorname{dim}_{F}\left(\mathcal{A}_{h}, H\right) \leq c_{5}$ where $c_{5}$ is the same constant (independent of $h$ ) as in Lemma 5.4.

\section{The MAIn CONVERGENCE RESUlt}

Up to now, we have only assumed that the family $\left(\mathcal{T}_{h}\right)_{h \in J}$ of triangulations is regular. For our final result, we will assume that this family of triangulations is quasi-uniform, i.e. it is regular and there exists a constant $\sigma_{1}>0$ such that

$$
\forall h \in J, \forall K \in \mathcal{T}_{h}, \quad h_{K} \geq \sigma_{1} h,
$$

where we use the notation of Section 3.1. This assumption allows us to use inverse estimates. In particular, we have:

Lemma 6.1. There exists a constant $M_{3}$ independent of $h$ such that

$$
\left\|P_{h} v\right\|_{V} \leq M_{3}\|v\|_{V}, \quad \forall v \in V .
$$

Proof. For $v \in V$, we have

$$
\begin{aligned}
\left\|P_{h} v\right\|_{V} & \leq\left\|\Pi_{h} v\right\|_{V}+\left\|\Pi_{h} v-P_{h} v\right\|_{V} \\
& \leq\|v\|_{V}+\left\|P_{h}\left[\Pi_{h} v-v\right]\right\|_{V}
\end{aligned}
$$

where we used (4.3) and $P_{h}\left[\Pi_{h} v\right]=\Pi_{h} v$. Since the family of triangulation is quasiuniform, we have the inverse estimate [10]

$$
\left\|w_{h}\right\|_{V} \leq \frac{C}{h}\left|w_{h}\right|_{H}, \quad \forall w_{h} \in V_{h},
$$

for some constant $C$ independent of $h$. On choosing $w_{h}=P_{h}\left[\Pi_{h} v-v\right]$, using (4.2) and (4.4), we find

$$
\left\|P_{h}\left[\Pi_{h} v-v\right]\right\|_{V} \leq \frac{C}{h}\left|\Pi_{h} v-v\right|_{H} \leq C M_{1}\|v\|_{V},
$$

and (6.1) follows with $M_{3}=1+C M_{1}$.

The error estimate with nonsmooth data can be modified as follows.

Theorem 6.2. For all $R_{1}>0$ and for all $T>0$, there exists a constant $C\left(R_{1}, T\right)$ independent of $h$ such that, if $\left\|u_{0}\right\|_{V} \leq R_{1}$ and $u_{h}^{0}=P_{h}\left(u_{0}\right)$, then

$$
\sup _{t \in[0, T]}\left|u_{h}(t)-u(t)\right|_{H} \leq C\left(R_{1}, T\right) h .
$$


Proof. Using Lemmas 2.5, 3.3 and 6.1, we find that

$$
\|u(t)\|_{V} \leq C_{1}\left(R_{1}\right) \quad \text { and } \quad\left\|u_{h}(t)\right\|_{V} \leq C_{1}\left(M_{3} R_{1}\right), \quad \forall t \geq 0 .
$$

As a consequence, $g(u)$ and $g\left(u_{h}\right)$ are bounded in $L^{\infty}\left(\mathbb{R}_{+} ; H\right)$, uniformly with respect to $h$. We may then argue exactly as in the proof of Theorem 4.6. We simply replace estimate (4.10) by

$$
\left|E_{h}(t) P_{h}\left(u_{0}\right)-E(t) u_{0}\right|_{H} \leq C R_{1} h, \quad \forall t \geq 0,
$$

which is a direct consequence of Proposition 4.3.

Our main result reads as follows.

Theorem 6.3. The continuous semigroup $\left\{S_{0}(t), t \in \mathbb{R}_{+}\right\}$on $H$ associated to $(2.1)$ possesses an exponential attractor $\mathcal{M}_{0}$ and for every $h \in J$, the continuous semigroup $\left\{S_{h}(t), t \in \mathbb{R}_{+}\right\}$on $V_{h}$ associated to (3.2) possesses an exponential attractor $\mathcal{M}_{h}$ such that:

(1) the fractal dimension of $\mathcal{M}_{h}$ is bounded, uniformly with respect to $h \in J$,

$$
\operatorname{dim}_{F}\left(\mathcal{M}_{h}, H\right) \leq c_{10},
$$

where $c_{10}$ is independent of $h$;

(2) $\mathcal{M}_{h}$ attracts the bounded sets of $V_{h}$, uniformly with respect to $h \in J$, i.e.

$\forall h \in J, \forall B_{h} \subset V_{h}$ bounded, $\operatorname{dist}_{H}\left(S_{h}(t) B_{h}, \mathcal{M}_{h}\right) \leq \mathcal{Q}\left(\|B\|_{H}\right) e^{-c_{11} t}, \quad t \geq 0$,

where the positive constant $c_{11}$ and the monotonic function $\mathcal{Q}$ are independent of $h$;

(3) the family $\left\{\mathcal{M}_{h}, h \in \bar{J}\right\}$ is continuous at 0 ,

$$
\operatorname{dist}_{\text {sym }}\left(\mathcal{M}_{h}, \mathcal{M}_{0}\right) \leq c_{12} h^{\kappa^{\prime}}
$$

where $c_{12}$ and $\kappa^{\prime} \in(0,1)$ are independent of $h$.

Proof. For every $h \in \bar{J}$, we construct $\mathcal{M}_{h}$ by the standard formula (see e.g. [5]):

$$
\mathcal{M}_{h}=\bigcup_{t \in[0, T]} S_{h}(t) \mathcal{M}_{h}^{d}
$$

where $T$ is defined by (5.1) and $\mathcal{M}_{h}^{d}$ is the exponential attractor from Lemma 5.4. The estimates from Sections 2 and 3 show that the function $F_{h}\left(t, v_{h}\right)=S_{h}(t) v_{h}$ is $1 / 2$-Hölder continuous for the time variable and Lipschitz continuous for the phase variable on $[0, T] \times \mathcal{B}_{h}$. This shows that $\mathcal{M}_{h}=F_{h}\left([0, T] \times \mathcal{M}_{h}^{d}\right)$ is compact and has a finite fractal dimension with

$$
\operatorname{dim}_{F}\left(\mathcal{M}_{h}, H\right) \leq 2+\operatorname{dim}_{F}\left(\mathcal{M}_{h}^{d}, H\right) \leq c_{5}+2 .
$$

A standard argument [5] shows that $\mathcal{M}_{h}$ is positively invariant, i.e. $S_{h}(t) \mathcal{M}_{h} \subset \mathcal{M}_{h}$ for all $t \geq 0$. From Property (2) of Lemma 5.4, we deduce that $\mathcal{M}_{h}$ attracts $\mathcal{B}_{h}$ exponentially, i.e.

$$
\operatorname{dist}_{H}\left(S_{h}(t) \mathcal{B}_{h}, \mathcal{M}_{h}\right) \leq C e^{-c_{11} t}, \quad t \geq 0,
$$

where the positive constants $C$ and $c_{11}$ are independent of $h$ (we may choose $c_{11}=$ $(\ln 2) / T)$. Using that $\mathcal{B}_{h}$ is an absorbing set in $V_{h}$, we obtain property (2) above. 
It remains to prove property (3). Let $\varepsilon>0$ and let $v_{0} \in \mathcal{M}_{0}$ be arbitrary. Then $v_{0}=S_{0}(t) w_{0}$ for some $t \in[0, T]$ and some $w_{0} \in \mathcal{M}_{0}^{d}$. By Lemma 5.4, there exists $w_{h} \in \mathcal{M}_{h}^{d}$ such that $\left|w_{0}-w_{h}\right|_{H} \leq c_{7} h^{\kappa}+\varepsilon / c_{4}$. We have, using Theorem 6.2,

$$
\begin{aligned}
\left|S_{0}(t) w_{0}-S_{h}(t) w_{h}\right|_{H} & \leq\left|S_{0}(t) w_{0}-S_{h}(t) P_{h} w_{0}\right|_{H}+\left|S_{h}(t) P_{h} w_{0}-S_{h}(t) w_{h}\right|_{H} \\
& \leq C\left(\mathcal{R}_{1}, T\right) h+c_{4}\left|P_{h} w_{0}-w_{h}\right|_{H} \\
& \leq C\left(\mathcal{R}_{1}, T\right) h+c_{4}\left|w_{0}-w_{h}\right|_{H} .
\end{aligned}
$$

In the last line, we used that $P_{h} w_{0}-w_{h}=P_{h}\left[w_{0}-w_{h}\right]$ and (4.2). Thus,

$$
\left|v_{0}-v_{h}\right|_{H} \leq\left(C\left(\mathcal{R}_{1}, T\right) h_{\text {max }}^{1-\kappa}+c_{4} c_{7}\right) h^{\kappa}+\varepsilon
$$

where $v_{h}=S_{h}(t) w_{h} \in \mathcal{M}_{h}$. Since $\varepsilon>0$ and $v_{0} \in \mathcal{M}_{0}$ are arbitrary, this shows that $\operatorname{dist}_{H}\left(\mathcal{M}_{0}, \mathcal{M}_{h}\right) \leq C h^{\kappa}$, where $C$ is independent of $h$. We prove similarly that $\operatorname{dist}_{H}\left(\mathcal{M}_{h}, \mathcal{M}_{0}\right) \leq C h^{\kappa}$. This yields Property (3) above.

Remark 6.4. As a consequence of Theorem 6.3, a solution of the semidiscrete problem gets close to the exponential attractor $\mathcal{M}_{0}$ in an exponential rate and remains in its neighborhood forever. This particular result was proved by Aida and Yagi for a finite element discretization of a reaction-diffusion system [1]. However, our result is much stronger because we estimate the symmetric Hausdorff distance between $\mathcal{M}_{h}$ and $\mathcal{M}_{0}$.

\section{ACKNOWLEDGEMENTS}

The author is thankful to Maurizio Grasselli and Madalina Petcu for stimulating discussions.

\section{REFERENCES}

[1] M. Aida and A. Yagi. Global stability of approximation for exponential attractors. Funkcial. Ekvac., 47(2):251-276, 2004.

[2] A. V. Babin and M. I. Vishik. Attractors of evolution equations, volume 25 of Studies in Mathematics and its Applications. North-Holland Publishing Co., Amsterdam, 1992.

[3] N. Batangouna and M. Pierre. Convergence of exponential attractors for a time splitting approximation of the Caginalp phase-field system. Commun. Pure Appl. Anal., 17(1):1-19, 2018.

[4] V. V. Chepyzhov and M. I. Vishik. Attractors for equations of mathematical physics, volume 49 of American Mathematical Society Colloquium Publications. American Mathematical Society, Providence, RI, 2002.

[5] A. Eden, C. Foias, B. Nicolaenko, and R. Temam. Exponential attractors for dissipative evolution equations, volume 37 of RAM: Research in Applied Mathematics. Masson, Paris; John Wiley \& Sons, Ltd., Chichester, 1994.

[6] M. Efendiev, A. Miranville, and S. Zelik. Exponential attractors for a nonlinear reactiondiffusion system in $\mathbf{R}^{3}$. C. R. Acad. Sci. Paris Sér. I Math., 330(8):713-718, 2000.

[7] M. Efendiev, A. Miranville, and S. Zelik. Exponential attractors for a singularly perturbed Cahn-Hilliard system. Math. Nachr., 272:11-31, 2004.

[8] C. M. Elliott and S. Larsson. Error estimates with smooth and nonsmooth data for a finite element method for the Cahn-Hilliard equation. Math. Comp., 58(198):603-630, S33-S36, 1992.

[9] C. M. Elliott and A. M. Stuart. The global dynamics of discrete semilinear parabolic equations. SIAM J. Numer. Anal., 30(6):1622-1663, 1993.

[10] A. Ern and J.-L. Guermond. Éléments finis: théorie, applications, mise en ouvre, volume 36 of Mathématiques \& Applications. Springer-Verlag, Berlin, 2002.

[11] P. Fabrie, C. Galusinski, and A. Miranville. Uniform inertial sets for damped wave equations. Discrete Contin. Dynam. Systems, 6(2):393-418, 2000. 
[12] P. Fabrie, C. Galusinski, A. Miranville, and S. Zelik. Uniform exponential attractors for a singularly perturbed damped wave equation. Discrete Contin. Dyn. Syst., 10(1-2):211-238, 2004.

[13] C. Galusinski. Perturbations singulières de problèmes dissipatifs : étude dynamique via l'existence et la continuité d'attracteurs exponentiels. PhD thesis, Université de Bordeaux, 1996.

[14] D. Gilbarg and N. S. Trudinger. Elliptic partial differential equations of second order. Classics in Mathematics. Springer-Verlag, Berlin, 2001.

[15] C. Johnson, S. Larsson, V. Thomée, and L. B. Wahlbin. Error estimates for spatially discrete approximations of semilinear parabolic equations with nonsmooth initial data. Math. Comp., 49(180):331-357, 1987.

[16] J.-L. Lions. Quelques méthodes de résolution des problèmes aux limites non linéaires. Dunod; Gauthier-Villars, Paris, 1969.

[17] A. Miranville, V. Pata, and S. Zelik. Exponential attractors for singularly perturbed damped wave equations: a simple construction. Asymptot. Anal., 53(1-2):1-12, 2007.

[18] A. Miranville and S. Zelik. Attractors for dissipative partial differential equations in bounded and unbounded domains. In Handbook of differential equations: evolutionary equations. Vol. $I V$, Handb. Differ. Equ., pages 103-200. Elsevier/North-Holland, Amsterdam, 2008.

[19] M. Pierre. Convergence of exponential attractors for a time semi-discrete reaction-diffusion equation. Numer. Math., Dec 2017.

[20] G. Raugel. Global attractors in partial differential equations. In Handbook of dynamical systems, Vol. 2, pages 885-982. North-Holland, Amsterdam, 2002.

[21] P.-A. Raviart and J.-M. Thomas. Introduction à l'analyse numérique des équations aux dérivées partielles. Collection Mathématiques Appliquées pour la Maîtrise. [Collection of Applied Mathematics for the Master's Degree]. Masson, Paris, 1983.

[22] J. Shen. Convergence of approximate attractors for a fully discrete system for reaction-diffusion equations. Numer. Funct. Anal. Optim., 10(11-12):1213-1234 (1990), 1989.

[23] A. M. Stuart and A. R. Humphries. Dynamical systems and numerical analysis, volume 2 of Cambridge Monographs on Applied and Computational Mathematics. Cambridge University Press, Cambridge, 1996.

[24] R. Temam. Infinite-dimensional dynamical systems in mechanics and physics, volume 68 of Applied Mathematical Sciences. Springer-Verlag, New York, second edition, 1997.

[25] V. Thomée. Galerkin finite element methods for parabolic problems, volume 25 of Springer Series in Computational Mathematics. Springer-Verlag, Berlin, second edition, 2006.

[26] X. Wang. Approximation of stationary statistical properties of dissipative dynamical systems: time discretization. Math. Comp., 79(269):259-280, 2010.

[27] X. Wang. Numerical algorithms for stationary statistical properties of dissipative dynamical systems. Discrete Contin. Dyn. Syst., 36(8):4599-4618, 2016.

[28] Y. Yan. Dimensions of attractors for discretizations for Navier-Stokes equations. J. Dynam. Differential Equations, 4(2):275-340, 1992.

[29] Y. Yan. Attractors and dimensions for discretizations of a weakly damped Schrödinger equation and a sine-Gordon equation. Nonlinear Anal., 20(12):1417-1452, 1993.

Laboratoire de Mathématiques et Applications, Université de Poitiers, CNRS, F86962 Chasseneuil, France. EMail: Pierre@math. univ-poititers. Fr 